\title{
Low socioeconomic status and visual health behaviors as factors in childhood myopia development
}

\author{
Cheng-Jong Lee ${ }^{1}$, Yu-Ting Hsiao ${ }^{1}$, Philip M. M. Sced ${ }^{2, a}$ and Shi-Huang Lee ${ }^{3}$ \\ ${ }^{1}$ Department of Sport and Health Management, Da-Yeh University, Taiwan \\ ${ }^{2}$ Department of English, Da-Yeh University, Taiwan \\ ${ }^{3}$ Department of Ophthalmology, Taichung Tzu Chi Hospital, Taiwan
}

\begin{abstract}
Although many studies have discussed visual acuity (VA), the childhood myopia epidemic has not been alleviated and requires further investigation. This study, therefore, investigates the differences in children's VA among various family structures and the effects of visual health attitudes (VHA) and visual health behaviors (VHB) on VA. The children in low socioeconomic status (SES) families in Changhua County, Taiwan are the main research subjects. Using purposive sampling, the study conducts a questionnaire survey and a VA examination on low SES children, undergoing counseling at two social welfare organizations in Taiwan. The VA examination was conducted at the two social welfare organizations by an ophthalmologist from Tzu Chi Hospital, Taichung, between October 2016 and December 2016. 265 questionnaires were returned. Significantly, over $60 \%$ of the samples of low SES children were not living with their parents. Of these children, the highest proportion (35.8\%) lived with their mothers; the second-highest (31.3\%), lived with their grandparents; and the third highest (29.4\%), lived with their fathers. $86.4 \%$ of the low SES children had poor (below 1.0) VA. The study, therefore, concludes that schools should focus on low SES students, and should focus on improving the VHA of the youngest students.
\end{abstract}

\section{Introduction}

The World Health Organization (WHO) estimates that there are 246 million visually impaired people worldwide and that $90 \%$ of these people are of low socioeconomic status (SES) [1]. According to the WHO, $43 \%$ of moderate to severe visual impairment is caused by uncorrected refractive errors, such as myopia, hyperopia, astigmatism and presbyopia. Although, the WHO reports that worldwide socioeconomic development and concerted public health action have brought about a general decrease in visual impairment worldwide, there is a scholarly consensus that myopia is increasing worldwide and is approaching epidemic proportions in the industrialized nations of East Asia [2-7]. Lee et al. [8] report a prevalence of myopia as high as $86.1 \%$ among young Taiwanese men aged 18 to 24 years.

Although myopia and other refractive error can be corrected with the aid of glasses, contact lenses and refractive surgeries, myopes will likely suffer to some degree from lost educational and employment opportunities, lost economic gain, and impaired quality of life [9, 10]. Moreover, myopes are at a higher risk of blindness in old age. The increased prevalence of myopia will lead to increases

\footnotetext{
${ }^{\mathrm{a}}$ Corresponding author : philsced@mail.dyu.edu.tw

(C) The Authors, published by EDP Sciences. This is an open access article distributed under the terms of the Creative Commons Attribution License 4.0 (http://creativecommons.org/licenses/by/4.0/).
} 
in the numbers of blind people, whose health care needs pose a serious socioeconomic burden to families and societies.

\subsection{Environmental factors}

Myopia has traditionally been considered a hereditary condition, and many previous studies indicate that children with myopia are more likely to have a family history of myopia [11-15]. However, there is a growing scholarly consensus that both environmental and hereditary factors play a role in the development of myopia. To cite a few of the studies that argue for the importance of environmental factors in myopia development, Xiang et al. found that a high percentage of children in Guangzhou become myopic by the age of 15, irrespective of the myopic status of their parents [16]. He et al. reported that, in China, school children in urban settings exhibit a higher prevalence of myopia than children in rural settings [17], suggesting that environmental factors are involved in the children's myopia development, because the genetic background of the children living in the urban and rural areas is similar. And Rose et al. found that a lower prevalence of myopia among Chinese children raised in Sydney than among Chinese children living in Singapore was associated with increased hours of outdoor activities [18].

\subsection{Reading habits and visual health behaviors}

One study [18] found that a lower prevalence of myopia among Chinese children raised in Sydney than among Chinese children living in Singapore was associated with increased hours of outdoor activities. Animal studies have shown that light levels as high as 15,000 to 30,000 lux either in the form of artificial laboratory light or natural daylight retard experimental myopia in chicks, guinea pigs and monkeys [10]. Based on these results from animal models, it is hypothesized that high light levels outdoors trigger the release of dopamine, an ocular growth inhibitor that inhibits myopic development.

Lyu et al. [4] investigated the reading habits, visual behaviors and living environments of elementary school children in Chaoyang District, Beijing and found that myopia was associated with reading posture, reading distance, and font size. You et al. [19] note that dim illumination during reading is an additional factor in the development of myopia. Does this association of reading habits and visual behaviors with myopia suggest that parents and teachers who promote healthy visual health behavior (VBH) in children may be able to help reduce the children's risk of myopia?

More research is needed to investigate whether unhealthy VHB such as not doing sufficient daytime outdoor activity, obsessively watching electronic devices (e.g. smartphones, computer monitors or TV screens) and continuously studying in dimly lit environments are factors in the development of myopia. Further study is also needed to investigate whether there is a relationship between unhealthy VHB and SES.

Previous studies have shown that high SES children exhibit higher incidences of myopia [19]. This study will focus on low SES children, investigating the healthiness of their VHB and probing the hypothesis that unhealthy VHB are causatives factor in myopia development. This study may, therefore, shed light on the question of whether parents who create healthy study environments and discipline their children to practice healthy VHB can reduce their children's risk of developing myopia.

\section{Materials and methods}

This study passed a review (CRREC-105-074) by the Institutional Review Board (IRB) of China Medical University Hospital, Taichung, Taiwan. The visual acuity (VA) examinations were conducted by an ophthalmologist from Tzu Chi Hospital, Taichung, Taiwan, between October 2016 and December 2016. Before any children were surveyed, both the children and their parents had signed a consent form, indicating that they understood the purpose and contents of the survey and that they were willing to answer the questions. The study was, therefore, carried out according to the 
regulations of the IRB. Children in low SES families counseled by three social welfare organizations in Taiwan were the research subjects. The research subjects included children who were in singleparent families, foster families, or families in which parental responsibility fell on grandparents, relatives, foreign spouses, and parents with physical or mental disorders.

\subsection{Research tools}

To anticipate the reliability of the questionnaire, a preliminary test using the questionnaire was conducted on students counselled by the social welfare organizations. A purposive sampling study and an examination of VA were also conducted as part of the preliminary study in October 2016. 50 questionnaires were returned in total. The test results indicated that both research variables were over .70 , showing a reliable result and a high interior consistency. 330 formal questionnaires were distributed in December 2016 and 265 questionnaires were returned. The scale reliability was above .70 , showing high consistency.

\section{Hypotheses}

Based on the widely utilized Theory of Planned Behavior, which suggests that people's behaviors can be predicted based on their attitudes [20], the purpose of this study was to investigate the relationship between VHB and visual health attitudes (VHA). The study's hypotheses follow.

1. The low SES children's VHA will exhibit a difference across demographic variables.

2. The low SES children's VHB will exhibit a difference across demographic variables.

3. VHA will have a positive impact on VHB.

4. VHB will have a positive impact on VA.

\section{Results}

\subsection{Samples structural analysis}

Structural analysis was carried out by SPSS 18.0. The demographic statistics in the basic data of the sample are shown by gender. There are 130 (49.1\%) males and $135(50.9 \%)$ females. 105 (39.6\%) students live with their father and mother and 160 (60.4\%) students do not, among whom, the highest proportion live with the mother (29.1\%). Significantly, of the samples of low SES children, over $60 \%$, therefore, do not live with their parents. Of the low SES children, the highest proportion (35.8\%) are raised by their mothers, the second highest proportion $(31.3 \%)$, by their grandparents, and the third highest (29.4\%), by their fathers. The proportion of children raised by their grandparents is, therefore, higher than the proportion raised by their fathers.

\subsection{VA testing}

Table 1. Eyes classified by unaided Visual Acuity (VA)

\begin{tabular}{cccc}
\hline VA & Case No. & Percentage & Accumulated Percentage \\
\hline Over 1.0 (normal) & 92 & 34.7 & 34.7 \\
0.8-0.9 (mild vision loss) & 26 & 9.8 & 44.5 \\
0.4-0.7 (ametropia) & 79 & 29.8 & 74.3 \\
Under 0.3 (Severe ametropia) & 68 & 25.7 & 100.0 \\
Total & 265 & 100.0 & \\
\hline
\end{tabular}

This study passed a review by the Institutional Review Board. The study conducted a questionnaire and performed a VA examination on low SES children. The VA examination used a standard visual 
scale to measure uncorrected defective VA. Any uncorrected VA less than 0.9 without other eye diseases was regarded as poor VA. The worst eye would be measured if both eyes exhibited poor VA, and the right eye would be measured if the eyes were of the same VA. The result of the examination is shown in table 1 . Approximately $65.3 \%$ of the student's VA is under 1.0 .

\subsection{Test of hypotheses}

The result of the ANOVA analysis, using school grades as the independent variable and VHB and VHA as dependent variables, showed that there was a significant difference $(p<.05)$ in VHA. As shown in Table 2, first graders scored lower than students in other grades in terms of "correct" VHA. The most probable reason for this low score is not the negligence of the teacher, but rather the naturally lower cognitive ability of the first graders, which impedes them from understanding the importance and benefits of visual health, despite the teacher's best efforts in the classroom. The visual health knowledge the first graders are taught does not turn into "correct" VHA. Therefore, families, schools and social welfare institutions should carefully consider how better to inculcate correct VHA in first graders, who are most in danger of losing at the start line.

Table 2. Single factor variance analysis of grade of students

\begin{tabular}{cccccc}
\hline Variable & Grade & Mean & $\begin{array}{c}\text { Standard } \\
\text { Deviation }\end{array}$ & F-value & PostHoc (Scheffe) \\
\hline & (1) First Grade & 2.190 & 0.722 & & \\
(2) Second Grade & 2.855 & 0.618 & & $(2)>(1)$ \\
Visual Health Attitude & (3) Third Grade & 2.712 & 0.644 & $5.169 *$ & $(4)>(1)(5)>(1)$ \\
(VHA) & (4) Fourth Grade & 2.881 & 0.629 & & $(6)>(1)$ \\
& (5) Fifth Grade & 2.793 & 0.590 & & \\
& (6) Sixth Grade & 2.923 & 0.481 & & \\
\hline
\end{tabular}

$* \mathrm{p}<.05$

A significant difference between the various family structures emerged regarding the questionnaire item "I have a balanced diet, and am not a picky eater". As shown in Table 3, the students who live with their father have a better-balanced diet than the students who live with their mother. A plausible explanation for this may be that fathers tend to discipline their children more strictly.

Table 3. Analysis of the differences between various family structures

\begin{tabular}{cccccc}
\hline Question & Family Structure & Mean & $\begin{array}{c}\text { Standard } \\
\text { Deviation }\end{array}$ & F-value & PostHoc (Scheffe) \\
\hline & (1) Live with parents & 2.619 & 0.944 & & \\
I have a balanced diet & (2) Live with father & 3.088 & 0.949 & \multirow{2}{*}{$4.694^{*}$} & $(2)>(3)$ \\
and am not a picky eater. & (3) Live with mother & 2.402 & 1.016 & & \\
& (4) Others & 2.605 & 1.027 & & \\
\hline
\end{tabular}

$* \mathrm{p}<.05$

As shown in Table 4, VHB and VHA exhibit no significant difference between male and female.

Table 4. Analysis of the differences between genders

\begin{tabular}{cccccc}
\hline Variable & Gender & Mean & Standard deviation & t-value & p-value \\
\hline \multirow{2}{*}{ Visual Health Attitude (VHA) } & male & 2.796 & 0.542 & .229 & .819 \\
& female & 2.778 & 0.694 & .22 & \\
\multirow{2}{*}{ Visual Health Behavior (VHB) } & male & 2.522 & 0.636 & \multirow{2}{*}{.682} & .496 \\
& female & 2.468 & 0.646 & & \\
\hline
\end{tabular}


The children whose VA was over 1.0 and between 0.4 to 0.7 had significant differences in 3 questions related to VHB, as shown in table 5. The children with good VA had established the habit of resting the eyes by taking a 10 -minute break after reading for 30 minutes, but the children whose VA was between 0.4 to 0.7 did not pay attention to resting the eyes, because they could see clearly, felt no myopia, and wanted to finish homework as soon as possible. The children with good VA had "correct" VHA and would do outdoor sports on weekdays and holidays. The low SES children did not have bright fluorescent lamps and table lamps, lived in poor environments and did not, in some cases, even have a desk. The majority of low SES children did not have a dedicated desk lamp and only turned on a lamp to write or read under weak light in order to save money, forcing them to maintain an unhealthy reading distance. The children whose VA was between 0.4 to 0.7 would squint their eyes to read, making it harder for the ciliary muscles of they eye to adjust. The children whose VA was between 0.4 to 0.7 could read clearly if they squinted their eyes and they did not think they had problems, because they experienced little adverse effect in their daily lives. They also tended to ignore VHB, feeling that wearing glasses made no difference to them, and that they did not have myopia.

Table 5. Analysis of the differences in visual health behavior (VHB) for various visual acuities (VA)

\begin{tabular}{clllll}
\hline \multicolumn{1}{c}{ VHB } & \multicolumn{1}{c}{ VA } & Vision Level & Mean & $\begin{array}{c}\text { Standard } \\
\text { deviation }\end{array}$ & $\begin{array}{c}\text { PostHoc } \\
\text { (Scheffe) }\end{array}$ \\
\hline A. I look into the distance and & $(1)$ over 1.0 & 2.625 & 1.099 & & \\
rest for 10 minutes after & $(2) 0.8-1.0$ & 2.313 & 1.026 & $2.994^{*}$ & $(1)>(3)$ \\
reading for 30 minutes. & $(3) 0.4-0.7$ & 2.012 & 1.006 & & \\
& $(4)$ under 0.3 & 2.236 & 0.980 & & \\
B. I spend at least 120 & $(1)$ over 1.0 & 2.968 & 1.031 & & \\
minutes outdoors every day. & $(3) 0.4-0.7$ & 2.525 & 1.003 & $4.814^{*}$ & $(1)>(3)$ \\
& $(4)$ under 0.3 & 2.189 & 1.013 & & \\
C. I maintain a reading & $(1)$ over 1.0 & 2.454 & 0.9588 & & \\
distance of over 35 cm while & $(2) 0.8-1.0$ & 2.606 & 0.999 & & \\
reading or doing homework. & $(3) 0.4-0.7$ & 2.303 & 1.078 & $3.695 *$ & \\
& $(4)$ under 0.3 & 2.436 & 0.976 & & \\
\hline
\end{tabular}

$* \mathrm{p}<.05$

\section{Discussion}

The assumptions in this study were analyzed using SPSS and AMOS statistical software, yielding the following results:

1. H1: The VHA of low SES children will exhibit a difference across the demographic variables.

As shown in Table 3, there was a significant difference between the school grades in regard to VHA; thus, H1 was feasible.

2. H2: The VHB of low SES children will exhibit a difference across the demographic variables.

As shown in table 4, there was a significant difference between various family structures in regard to VHB (e.g. not a picky eater); thus, $\mathrm{H} 2$ was feasible.

3. H3: Student's VHA will have a positive impact on VHB.

As shown in Table 5, the regression coefficient of VHA to VA was .406, achieving a significance level of $\mathrm{p}<.05$; thus, H3 was feasible. B5, "I take a rest instead of seeing an ophthalmologist when I can't see clearly," was the most effective question regarding VHA (regression coefficient .746).

4. H4: VHB will have a positive impact on VA.

As shown in Table 5, there was a significant difference $(\mathrm{p}<.05)$ in regression coefficient $(.224)$ of VHB to VA; thus, H4 was feasible, and VHB had a positive impact on VA. C2 "I look into the distance and rest for 10 minutes after reading, watching $\mathrm{TV}$, playing games, playing smartphone or 
computer for 30 minutes" was the most effective question regarding VHB (regression coefficient .657).

$65.3 \%$ of the low SES children's vision was below 0.9, which is an increase of $19.18 \%$ on the defective vision rate in Taiwan (46.12\%). A failure to maintain a healthy book-to-eye distance when reading seemed to be the main cause of myopia onset. The children with poor VA had worse VHA, leading to unhealthy VHB and ultimately to myopia. A further cause of myopia onset might be the ubiquity of addictive electronic devices such as smartphones and tablets. Los SES parents or guardians be too busy working to take care of their children, who consequently do not develop adequate self-control to use such electronic devices responsibly. In some cases, the children may even be encouraged to spend excessive amounts of time passively watching these devices, a phenomenon known informally in Taiwan as " $3 \mathrm{C}$ babysitting". This phenomenon may also occur in families where children are raised by grandparents, who are less likely to be strict about visual health. Families with foreign spouses also tend to be low SES and, therefore, may be too busy working to care for their children or may resort to "grandparenting" or even "3C babysitting".

\section{Findings}

According to the results of the study, "correct" VHA led to "correct" VHB, and correct VHB ensured good VA for students. Although myopia and other ametropias can be corrected by glasses and refractive surgery, myopia still causes a certain loss of education and employment opportunities as well as economic loss and general loss of quality of life $[9,10]$. Low SES children need more concern from parents, guardians and schools, in addition to better visual conditions to facilitate the reversal of their low SES. It is also expected that ophthalmologists, optometrists, and social workers will be more active in assisting with the visual health care of low SES children.

\section{Implications}

The results showed that junior students had bad VHA, implying there is still a deficiency in both the quality and quantity of visual health education in schools. To guide the next generation of students and parents towards deeply-rooted and correct VHA, schools should be active in providing effective, understandable, multi-language visual health guidelines for junior students and their families.

\section{Limitations}

1. Because the questionnaire and VA examination needed to have a parent or guardian's signature and the students' consent before filling in and testing, it was not possible to obtain test results from all the low SES elementary school students in Changhua County.

2. A purposive sampling study was conducted on the results of the VA examination of the low SES children who were counseled by social welfare organizations in Changhua County. We were not able to collect the data for those who were not counseled by social welfare organizations.

3. Because a guidance counselor read the questions to and helped each of the children complete the questionnaire, it is possible that error crept into the results due to the children's being unduly influenced by the counselor or to the children's failing to comprehend the questions.

\section{Suggestions for future research}

The VA examination and questionnaire were conducted using quantitative research, which required the respondents to answer on a scale of 1 to 4 . The respondents might have had reservations about or compromised or even randomly filled in their answers. There are few studies on the visual health care of low SES children and, as a result, there was little literature and few study results that could be verified or analyzed. There is a need for more research on myopia among low SES children generally, 
but there is also a lack of research using qualitative methods to study children with poor VA, obtaining data through in-depth interviews.

\section{References}

1. World Health Organization, http://www.who.int/mediacentre/factsheets/fs282/en/ (Accessed 21 Jan 2017)

2. L.L.K. Lin, Y.F. Shih, C.K. Hsiao and C.J. Chen, Annals Academy of Medicine Singapore, 33(1), 27-33 (2004)

3. S. Vitale, R.D. Sperduto and F.L. Ferris, Arch Ophthalmol, 127(12), 1632-1639 (2009)

4. Y. Lyu, H. Zhang, Y. Gong, D. Wang, T. Chen, X. Guo, S. Yang, D. Liu and M. Kang, China. Jpn J Ophthalmol, 59, 421-429 (2015)

5. D.C. Tsai, L.J. Lin, N. Huang, C.C. Hsu, S.Y. Chen, A.W.H. Chiu and C.J.L. Liu, Clin Exp Ophthalmol, 43, 612-620(2015)

6. I. G. Morgan, K. Ohno-Matsui and S.M. Saw, Myopia. Lancet., 379, 1739-1748 (2012)

7. P.C. Wu, C.L. Tsai, H.L. Wu, Y.H. Yang and H.K. Kuo, Ophthalmology, 120, 1080-1085 (2013)

8. Y.Y. Lee, C.T. Lo, S.J. Sheu and L.T. Yin, Ophthalmic Epidemiol, 22, 66-73 (2015)

9. B. Holden, P. Sankaridurg, E. Smith, T. Aller, M. Jong and M. He, Eye, 28, 142-146 (2014)

10. D. Ramamurthy, S.Y. Lin Chua and S.M. Saw, Clin Exp Optom, 98, 497-506 (2015)

11. L.T. Lim, Y. Gong, E. Y. Ah-kee, G. Xiao, X. Zhang and S. Yu, Ophthalmol Eye Dis, 6, 31-35 (2014)

12. I. Morgan and K. Rose, Prog Retin Eye Res., 24, 1-38 (2005)

13. D.O. Mutti, G.L. Mitchell, M.L. Moeschberger, L.A. Jones and K. Zadnik, Invest Ophthalmol Vis Sci., 43, 3633-3640 (2002)

14. S.M. Saw, J. Katz, O.D. Schein, S.J. Chew and T.K. Chan, Epidemiol Rev., 18, 175-187 (1996)

15. D.O. Mutti, K. Zadnik and A.J. Adams, Invest Ophthalmol Vis Sci., 37, 952-957 (1996)

16. F. Xiang, M. He and I.G. Morgan, Optom Vis Sci., 89, 1487-1496 (2012)

17. M. He, Y. Zheng and F. Xiang, Optom Vis Sci., 86, 40-44 (2009)

18. K. A. Rose, I.G. Morgan, W. Smith, G. Burlutsky, P. Mitchell and S.M. Saw, Arch Ophthalmol, 126, 527-530 (2008)

19. Q.S. You, L.J. Wu, J.L. Duan, Y.X. Luo, L.J. Liu, X. Li, Q. Gao, W. Wang, L. Xu, J.B. Jonas and X.H. Guo, PLoS One, doi: 10.1371/journal.pone.0052668 (2012)

20. M. Fishbein and I. Ajzen, Predicting and Changing Behavior: The Reasoned Action Approach (New York: Taylor \& Francis, 2010) 